\title{
Is Agency Skin Deep? Surface Attributes Influence Infants' Sensitivity to Goal-Directed Action
}

\author{
Jose J. Guajardo and Amanda L. Woodward \\ Department of Psychology \\ University of Chicago
}

\begin{abstract}
Three studies investigated the role of surface attributes in infants' identification of agents, using a habituation paradigm designed to tap infants' interpretation of grasping as goal directed (Woodward, 1998). When they viewed a bare human hand grasping objects, 7- and 12-month-old infants focused on the relation between the hand and its goal. When the surface properties of the hand were obscured by a glove, however, neither 7- nor 12-month-old infants represented its actions as goal directed (Study 1). Next, infants were shown that the gloved hands were part of a person either prior to (Study 2) or during (Study 3 ) the habituation procedure. Infants who actively monitored the gloved person in Study 2 and older infants in Study 3 interpreted the gloved reaches as goal directed. Thus, varying the extent to which an entity is identifiable as a person impacts infants' interpretation of the entity as an agent.
\end{abstract}

The distinction between animate agents and inanimate objects is fundamental to both everyday and abstract acts of human cognition. Observing a child chasing a soccer ball, we are not surprised when the child swerves to avoid a tree, but we would be if the ball behaved like the child. We identify the child as an animate agent, and therefore interpret her behavior as expressing her underlying goals or intentions. We understand that this interpretation is not appropriately extended to inanimate objects such as balls. Our ability to quickly categorize entities as agents or inanimate objects is supported by rich perceptual regularities. Animate agents differ from inanimate objects in their typical shapes, rigidity, surface properties, sounds, and patterns of motion. These perceptual attributes correlate with the behavioral and psychological attributes that are central to folk conceptions of agents

Requests for reprints should be sent to Amanda L. Woodward, Department of Psychology, University of Chicago, 5848 South University Avenue, Chicago, IL 60637. E-mail: woodward@uchicago.edu 
and thereby serve as concrete indicators of the unobservable, yet conceptually central, qualities of agents.

In this article, we investigate the state of this fundamental distinction during infancy. Recent findings indicate that during the first year of life, infants begin to interpret some observed behaviors not as purely physical motions through space but instead as goal-directed actions (Csibra, Gergely, Biro, Koos, \& Brockbank, 1999; Gergely, Nadasdy, Csibra, \& Biro, 1995; Legerstee, Barna, \& DiAdamo, 2000; Woodward, Sommerville, \& Guajardo, 2001). Thus, infants seem to have some knowledge about the core attributes of agents, in particular, their goal directedness. This then raises the question of how infants first identify the class of entities whose motions are properly understood as goal directed.

One well-known answer to this question is that infants initially detect potential agents based exclusively on their patterns of motion. Several related theories start from the assumption that infants are innately endowed with abstract conceptions of intentionality and then propose that these abstract systems are triggered by particular patterns of motion. Although there are different variants of this position, they converge in predicting that infants will initially attribute agency to a broad range of entities, so long as the entity moves in the right way. To illustrate, in an influential theoretical paper, Premack (1990) proposed that if an entity exhibits self-propelled motion, then infants will perceive it as a goal-directed agent solely on these grounds. This proposal was elaborated by Baron-Cohen (1995), who broadened the definition of self-propelled motion to include biological motion. He proposed that

the visual input might look as shapeless as an ameba, as weird as a giraffe, or as minimal as a stick insect. Because of their self-propelled motion, all these are instantly interpretable [by infants] as agents with goals and desires. (p. 34)

Gergely, Csibra, and their colleagues put forth a related but importantly distinct formulation (Csibra, Biro, Koos, \& Gergely, 2003; Csibra et al., 1999; Gergely et al., 1995; see also Király, Jovanovic, Prinz, Aschersleben, \& Gergely, 2003). They proposed that infants interpret observed events as goal directed based on a system for detecting rational patterns of motion with respect to a goal. This system is activated whenever infants view motion that can be interpreted as the most efficient route to a goal given situational constraints, regardless of the appearance of the moving entity. That is, infants are argued to "apply a psychological interpretational system which represents the observed behaviors in teleological terms ... and do so without any a priori ontological commitment concerning the types of objects which exhibit these behaviors" (Csibra et al., 1999, p. 261). Recently, Király et al. summarized this general position as follows:

Several theories propose innately based, abstract, and domain-specific representational systems specialized for identifying intentional agents ... While these models 
differ in several important respects, they all assume an initially wide scope of entities ... that infants can recognize as goal-directed from very early on (including unfamiliar actions of humans or unfamiliar agents with no human features). This generality in scope is due to the fact that these theories all postulate or imply sensitivity to abstract behavioural cues ... that indicate agency ... irrespective of previous experience with the types of agents or actions that exhibit these cues. (p. 753)

Consistent with these proposals, there is evidence that by 12 months of age, infants respond to unusual entities as if they were agents in some situations. Much of this evidence comes from the programmatic studies conducted by Gergely, Csibra, and their colleagues. Across several experiments, these researchers report that 12-month-old, and sometimes 9-month-old, infants view the motions of two-dimensional animated shapes in accord with the rationality principle - that is, the belief that agents seek efficient paths to reach goals (Csibra et al., 2003; Csibra et al., 1999; Gergely et al., 1995). To illustrate, in one study (Gergely et al., 1995) 12-month-old infants were habituated to a repeating scene in which a shape took a circuitous path around a barrier to reach another shape (the "goal"). Then, infants were shown test events in which the barrier had been removed and the shape moved either in the same circuitous path to reach the goal (irrational motion) or in a straight line to the goal (rational motion). Infants looked longer at the former test event than the latter, despite the fact that it repeated the same path of motion as during habituation. Gergely, Csibra, and colleagues concluded that infants interpreted the circuitous path as irrational, and therefore surprising, given the removal of the barrier. In addition to these findings, other researchers have reported that 12- to 15-month-old infants respond to novel objects or animated shapes as if they were agents when these entities behave in a manner designed to be consistent with this interpretation-for example when these entities respond contingently to social partners (S. Johnson, Booth, \& O’Hearn, 2001; S. Johnson, Slaughter, \& Carey, 1998; Kuhlmeier, Wynn, \& Bloom, 2003b; Shimizu \& Johnson, 2004).

Based on these findings it might be concluded that infants are different from adults in their identification of agents, relying not on the rich perceptual attributes of real-world agents but instead attending only to the patterns of motion that specify agency. To illustrate, imagine (a) a snake lying still and straight across a hiking trail, and (b) a blue plastic sphere rolling on an irregular trajectory over and around objects in its path, apparently in pursuit of another sphere. To a mature observer, the first of these is automatically identified as an animate agent, despite its lack of motion. Moreover, no matter how complex the behavior of the second, mature observers would never classify it as an agent. The theories and findings just described suggest that infants would, at least initially, make the opposite attributions.

To be clear, the relevant point is not whether or not motion provides information about agency, but rather, whether motion is the sole source of evidence considered in determining whether an entity is an agent. Adults certainly use patterns of mo- 
tion as one source of information about agency, and they might identify an entity as an agent based on its movements in the absence of other featural information, for example, when an indistinct entity is seen to move through the night. However, the claim is that infants attribute agency based exclusively on patterns of motion regardless of the other perceptual evidence available to them. Indeed, infants have been argued to respond to entities that have almost no attributes in common with real-world agents (e.g., two-dimensional geometrical shapes on a computer screen) as if they were agents.

This claim seems at odds with other evidence. For one, from the earliest months of life, infants are sensitive to the rich perceptual attributes of real-world agents (in particular, people), including facial configurations (M. Johnson, 1992), biological patterns of motion (Bertenthal, 1993), and the sounds they produce (Spelke, 1976). Moreover, infants connect these perceptual attributes to their developing knowledge of how agents behave. For example, by 6 months of age, infants expect people, but not inanimate objects, to engage in self-propelled motion and conversational exchanges (e.g., Kosugi \& Fujita, 2002; Legerstee et al., 2000; Molina, Van de Walle, Condry, \& Spelke, in press; Poulin-Dubois, Lepage, \& Ferland, 1996; Spelke, Phillips, \& Woodward, 1995; for a review, see Rakison \& Poulin-Dubois, 2001).

Further, infants as young as 6 months of age differentiate between people and inanimate objects in paradigms designed to assess their sensitivity to the goal directedness of actions. To illustrate, in one study (Woodward, 1998), infants viewed events in which a person reached toward and grasped one of two objects. To a mature observer this action is most readily described in terms of the relation between agent and goal ("she grasped the bear") rather than in terms of the strictly physical properties of the person's motion. That is, this action is interpreted as being goal directed. Infants were habituated to one reaching event, and then presented with test events that varied either the relation between actor and goal while preserving other aspects of the actor's motion (new object events), or preserved the relation between actor and goal while varying the physical properties of the actor's reach (new side events; see Figure 1). If infants represented the habituation event in terms of the actor-goal relation, then they were predicted to look longer on test events that vary this relation than on events that preserve it. Infants as young as 5 to 6 months of age responded in this way, so long as the actor they observed was a person. When they viewed inanimate "actors," such as rods, arm-shaped occluders, or mechanical claws, infants did not respond in this way. Subsequent findings further support the conclusion that infants interpret human grasps but not the grasps of inanimate claws as being goal directed, even in the potentially more informative context of seeing the claw move the object (Jovanovic et al., 2003). Thus, during the first year of life, infants analyze certain human actions in terms of their goal structure, but they seem not to readily extend this interpretation to the similar motions of inanimate objects. 


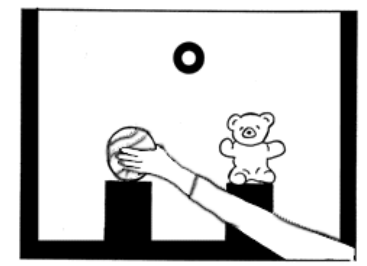

Habituation Event

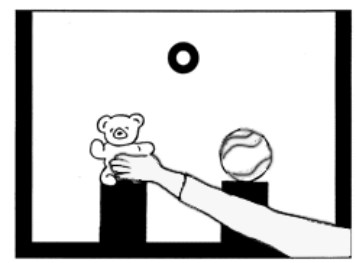

New-Object Event

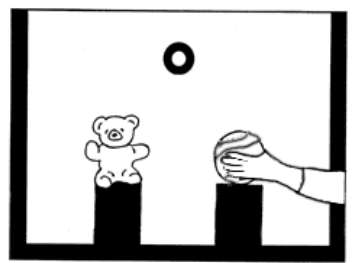

New-Side Event

FIGURE 1 Sample habituation and test events for Experiments 1 and 2.

These findings suggest a very different conclusion from the position described earlier: Rather than broadly attributing agency based on abstract patterns of motion, infants seem to draw on their knowledge of the attributes of familiar agents. These studies were designed to more rigorously test this possibility. In the Woodward (1998) studies, the human and inanimate actors differed on many dimensions, including the presence or absence of biological motion. Given Baron-Cohen's (1995) suggestion that biological motion could be considered a type of self-propelled motion, it is possible that this featural difference accounted for the difference in infants' interpretation of the human and inanimate actors' motions. To test this possibility, we presented infants with agents that were equated in terms of their motion properties but varied on a dimension that is diagnostic of animacy from a mature perspective, namely, surface texture. Surface properties are strong cues to animacy for adults—recall the snake lying across the trail—and infants are also sensitive to the surface properties that are typical of animate and inanimate objects (Smith \& Heise, 1992).

We adapted the method developed in Woodward (1998), this time presenting all infants with events in which a person grasped one of two toys with her hand. We varied the surface texture of the hand by having the presenting experimenter wear a metallic glove. In Study 1, infants viewed events like those depicted in Figure 1only the actor's arm and hand were visible. One group of infants (the bare hand condition) saw the actor's bare hand and the other (the gloved hand condition) saw it gloved. In all other respects - the biological patterns of motion, the shape of the hand, and the way in which the fingers articulated on the object-the two events were identical. In terms of self-propelledness, the two events were equally ambig- 
uous. Because the arm entered from the side of the stage, there was no evidence, beyond the biological nature of the arm's motion, that the arm was self-propelled. Thus, if infants rely on patterns of motion to identify agents, they would be predicted to represent the grasping events as goal-directed in both conditions. However, if infants are influenced by the typical features of agents, then altering the surface properties of the hand, particularly when only the hand itself is visible, might disrupt infants' interpretation of the grasping events as goal-directed.

We tested infants at two ages, 7 and 12 months, because the first of these approximates the younger ages tested by Woodward (1998), and the second approximates the ages tested in studies reporting breadth in infants' attributions of agency.

\section{STUDY 1}

\section{Method}

Participants. Sixty-four infants from a large metropolitan area participated in Study 1. The infants were all full term (at least 37 weeks gestation). Their parents had been contacted through mailings and advertisements and were offered $\$ 10$ to reimburse them for their travel expenses. The 32 infants in the 7-month age group ranged in age from 5 months, 26 days to 8 months, 9 days ( $M=7$ months, 11 days). The 32 infants in the 12-month age group ranged in age from 11 months, 1 day to 13 months, 7 days ( $M=11$ months, 27 days). At each age, 16 infants saw an actor grasp a toy with a gloved hand (gloved hand condition), and 16 infants saw her grasp a toy with her bare hand (the bare hand condition). At 12 months there were 7 girls and 9 boys in the gloved hand condition and 8 girls and 8 boys in the bare hand condition. At 7 months there were 8 girls and 8 boys in the gloved hand condition and 6 girls and 10 boys in the bare hand condition. Thirteen additional infants began the procedure but were excluded from the final sample due to failure to complete all trials (three 7-month-olds and four 12-month-olds) or because of an error in the procedure (one 7-month-old and five 12-month-olds).

Procedure. Sitting in either an infant seat or on a parent's lap, the infants faced a stage approximately $30 \mathrm{in} .(76.2 \mathrm{~cm})$ away on which two toys were positioned on 10-in.-tall $(25.4 \mathrm{~cm})$ pedestals that stood approximately $10 \mathrm{in} .(25.4 \mathrm{~cm})$ apart. One toy was a white teddy bear and the other was a multicolored ball. The back and sides of the stage were draped in black fabric. Infants were filmed by a video camera that was mounted between the toys with its lens protruding through the back curtain. Between trials, a white screen was raised to block the stage and toys from the infant's view.

During habituation, infants viewed the actor reach for the same toy, in the same location, on each trial (see Figure 1). In both conditions, the infants saw only the actor's arm and hand, and the actor's arm was covered in a red sleeve. In the gloved 
hand condition, the actor wore a metallic gold glove that covered all traces of her skin. In the bare hand condition, she did not wear the glove. At each age and in each condition 8 infants saw the actor reach to the right side of the stage, except in the 7-month bare hand condition, in which 7 infants saw the actor reach to the right side of the stage. At 12 months, 8 infants in each condition saw the actor grasp the bear during habituation. At 7 months, 9 infants in the gloved hand condition and 10 infants in the bare hand condition saw her grasp the bear.

An observer coded the infant's looking online from a video monitor, pressing a computer key when the infant was looking at the area containing the two toys and the actor's arm. The observer could not see the experimental events and did not know which condition was being presented. A computer program calculated the total looking time for each trial and determined when the infant had met the habituation criterion (Pinto, 1994). The infant's looking was coded beginning when the actor's hand made contact with the toy. This was accomplished by having another experimenter watch the event and signal the computer to begin timing at the appropriate point.

The trial ended when the infant looked away for 2 consecutive sec or when 120 sec had elapsed. The habituation criterion was calculated using the first three trials that totaled to at least $12 \mathrm{sec}$. When the infant's looking times on three consecutive trials were less then $50 \%$ of the average of the first three trials, the habituation phase ended. If the infant did not meet the habituation criterion after 14 trials, the habituation phase was ended and the test trials began.

Immediately after habituation, the toys' positions were reversed while the screen blocked the infant's view. The screen was then lowered and infants received one familiarization trial on which the actor did not reach into the stage to introduce them to the toys' new positions.

After the familiarization trial, infants saw two kinds of test events in alternation, for a total of three trials of each type. On new object trials, the actor reached to the same side of the stage as during habituation, now grasping the other toy. On new side trials, the actor reached to the other side of the stage to grasp the same toy as during habituation. At each age and in each condition, 8 infants began the test phase with a new side trial except in the 7-month gloved hand condition, in which 9 infants began with a new side trial.

Reliability coding. To assess reliability, a second independent observer coded each infant from the videotape. The two observers were strongly correlated in their judgments of looking times on the two kinds of test trials $(r=.96)$. Because this correlation could be inflated by the fact that the first observer determined when the trial ended, we undertook a second, more conservative analysis comparing the two observers' judgments of the endpoint for each trial. The online and video observers were scored as agreeing if they judged the trial to end at the same time, as indicated by there being no gap between the computer tone signaling the end of the 
TABLE 1

Attention During Habituation Trials and the Three Test Trials of Each Type for Each Age Group in Study 1

\begin{tabular}{|c|c|c|c|c|c|c|c|c|c|c|}
\hline \multirow{4}{*}{$\begin{array}{l}\text { Age Group and } \\
\text { Condition }\end{array}$} & & & \multicolumn{8}{|c|}{ Average Looking Time (in Sec) } \\
\hline & \multirow{2}{*}{\multicolumn{2}{|c|}{$\begin{array}{c}\text { No. of } \\
\text { Habituation } \\
\text { Trials }\end{array}$}} & \multicolumn{4}{|c|}{ Habituation } & \multicolumn{4}{|c|}{ Test Trials } \\
\hline & & & \multicolumn{2}{|c|}{ First 3} & \multicolumn{2}{|c|}{ Last 3} & \multicolumn{2}{|c|}{ New Side } & \multicolumn{2}{|c|}{ New Object } \\
\hline & $M$ & $S E$ & $M$ & $S E$ & $M$ & $S E$ & $M$ & $S E$ & $M$ & $S E$ \\
\hline \multicolumn{11}{|l|}{7 months } \\
\hline Bare hand & 8.4 & 0.7 & 16.8 & 2.5 & 7.8 & 1.9 & 6.1 & 0.9 & 8.7 & 1.6 \\
\hline Gloved hand & 7.9 & 0.6 & 19.7 & 3.7 & 5.9 & 0.8 & 9.3 & 2.8 & 6.9 & 1.3 \\
\hline \multicolumn{11}{|l|}{12 months } \\
\hline Bare hand & 9.8 & 0.8 & 8.8 & 1.1 & 5.4 & 1.4 & 5.4 & 0.9 & 8.1 & 1.3 \\
\hline Gloved hand & 8.7 & 0.7 & 13.7 & 2.6 & 3.8 & 0.7 & 6.0 & 0.9 & 6.8 & 0.8 \\
\hline
\end{tabular}

trial for the video observer and the tone recorded on the tape for the online observer. The tone was $0.2 \mathrm{sec}$ in duration. Thus, when observers agreed, they were within $0.2 \mathrm{sec}$ in their estimation of the trial ending. The observers agreed on $91 \%$ of the trial endings for 7-month-olds and $93 \%$ for 12-month-olds. To assess the possibility of observer bias, the disagreements were classified as early (the online observer ended the trial earlier than the video observer) or late (the online observer ended the trial later than the video observer) and as occurring on new object test trials or new side test trials. The disagreements were distributed randomly across these categories at both ages and in both conditions, all Fisher's exact $p>.99$.

\section{Results}

Table 1 summarizes infants' attention during habituation and test trials. An initial concern was whether there would be an overall difference in infants' interest in the gloved and ungloved hands. One way of investigating this possibility is to compare attention during habituation in the two conditions. A repeated measures analyses of variance (ANOVA) with condition (gloved hand vs. bare hand) and age group (7 months vs. 12 months) as between-subjects factors was conducted on infants' looking times on the first and last three habituation trials. ${ }^{1}$ This analysis revealed main effects for trial, $F(5,300)=36.30, p<.0001$, indicating a decline in looking across the habituation phase; and for age group, $F(1,60)=6.70, p<.05$, reflecting longer looking times on the part of 7 -month-olds, and no other reliable effects. No-

\footnotetext{
${ }^{1}$ There was positive skew in the habituation data for this study and for Studies 2 and 3. For this reason, all analyses involving habituation data were conducted after the data were subjected to a $\log$ transformation.
} 
tably, there was not a reliable main effect of condition, $F(1,60)=0.03, p>.8$. A similar analysis conducted on the number of trials required to meet the habituation criterion revealed no reliable effects. Thus, these analyses did not indicate that infants differed in their overall levels of attention to the gloved and bare hands. Four infants in the 12-month bare hand group and 1 in each of the other three groups reached 14 habituation trials without meeting criterion.

The principle analyses concerned infants' responses on the two kinds of test trials. Preliminary analyses revealed no significant effects of the sex of the infant, the order of test trials, the side of reach during habituation, or the object grasped during habituation. Therefore, subsequent analyses collapsed across these dimensions. Infants' looking times were averaged across the three trials of each type, and a repeated measures ANOVA was conducted on these scores with condition (gloved hand vs. bare hand) and age group (7 months vs. 12 months) as the between-subjects variables and trial type (new object vs. new side) as the within-subjects variable. This analysis revealed a Condition $\times$ Trial Type interaction, $F(1,60)=7.60, p<.01$, indicating that infants in the two conditions responded differently on the two kinds of test trials, and no other reliable effects. ${ }^{2}$ Planned contrasts revealed that infants in the bare hand condition looked longer on new object trials than on new side trials, $t(31)=3.29, p<.005$, but infants in the gloved hand condition did not differ in their attention to the two kinds of test trials, $t(31)<1$. There was no indication that this pattern varied as a function of age; neither the main effect nor any interactions involving age approached statistical significance, all $p \mathrm{~s}>.19$. The absence of a significant main effect of condition, $F(1,60)=0.025, p>.8$, further indicated that infants were equally attentive to the bare and gloved hands.

Although the ANOVA did not indicate reliable differences between the two age groups, follow-up analyses were conducted to assess whether each age group reflected the pattern shown by the sample as a whole. Paired comparisons of infants' looking times on new object and new side trials were conducted for each age group within each condition. ${ }^{3}$ These analyses indicated that neither age group differentiated between the two kinds of test trials in the gloved hand condition, $t(15)=1.10$, $p>.2$ at 12 months, and $t(15)=0.71, p>.49$ at 7 months. In the bare hand condition, 12-month-olds looked reliably longer on new object trials than new side trials, $t(16)=2.86, p<.05$, and 7 -month-olds showed a trend in the same direction, $t(15)=1.88, p<.08$. This trend reached significance when the infant who did not meet the habituation criterion was excluded from the analysis, $t(14)=2.20, p<.05$.

\footnotetext{
${ }^{2}$ The data from the 7-month-olds had positive skew. When the data were log transformed, to reduce this skew, the results of the Age Group $\times$ Condition $\times$ Trial Type ANOVA were similar to those reported for the untransformed scores: There was a main effect of trial type, $F(1,60)=6.12, p<.05$, qualified by a Condition $\times$ Trial Type interaction, $F(1,60)=4.55, p<.05$, and no other reliable effects.

${ }^{3}$ The data for the 7 -month-olds were first log transformed to reduce positive skew.
} 
Excluding nonhabituators did not alter the findings for the 12-month-olds or for the 7-month-olds in the gloved hand condition.

A secondary question concerns whether infants recovered attention during test trials relative to the end of habituation. Both new side and new object test trials presented infants with new information - the toys were in new positions on both kinds of trials, the arm reached to a new location on new side trials, and a new object was grasped on new object trials. To further investigate infants' attention to these changes, planned contrasts compared infants' average looking times on each kind of test trial to their average looking times on the last three habituation trials. This analysis strategy, although potentially informative, should be evaluated with caution. Because the final habituation trials are selected to be lower than others, across the board recovery is difficult to interpret. Moreover, because attention tends to decline across the experimental session (infants habituate to all aspects of the experiment), across the board failure to recover is also difficult to interpret. Twelvemonth-olds in the bare hand condition recovered on new object trials, $t(15)=2.89$, $p<.05$, but not on new side trials, $t(15)=0.79, p>.4$. This pattern is consistent with prior findings in suggesting that despite the new elements in new side trials, infants did not show a strong novelty response on these trials (see Woodward, 1998, 2003). In the gloved hand condition, 12-month-olds recovered on both kinds of test trials, $t(15)=3.35, p<.05$ for new object trials, and $t(15)=3.53, p<.05$ for new side trials. Seven-month-olds did not show reliable recovery in either condition for either type of test event: In the bare hand condition, $t(15)=1.22, p>.2$ for new object trials, and $t(15)=-0.16, p>.8$ for new side trials; in the gloved hand condition, $t(15)=0.94, p>.3$ for new object trials, and $t(15)=1.25, p>.2$ for new side trials.

\section{Discussion}

When they viewed grasping by a bare hand, 7- and 12-month-old infants selectively encoded the relation between the actor and the object she grasped. Having been habituated to one grasping action, infants looked longer on test trials that disrupted the relation between actor and object than at test trials that disrupted her patterns of motion while preserving this relation. These results replicate prior findings (Jovanovic et al., 2003; Woodward, 1998, 1999, 2003) and further support the conclusion that infants interpret human grasps as goal directed during the first year of life. In contrast, infants who saw the grasp performed by a gloved hand did not respond systematically on test trials. This difference between the two conditions seemed not to be a product of infants' finding the glove distracting or aversive; across both habituation and test trials infants were equally attentive to the gloved and bare hand events. There was no indication of a difference between the two age groups in the overall analysis, and the two age groups showed comparable patterns when analyzed separately. These findings suggest that the typical surface features 
of agents are relevant to infants' determination that an action is goal-directed at both 7 and 12 months.

In contrast to the proposal that infants identify a broad range of entities as potential agents based only on a small set of motion cues, the findings of Study 1 indicate that infants also rely on other kinds of featural support to identify agents. To adult eyes, the gloved hands were readily identifiable as the hands of a personthey were shaped like hands, moved like hands, and articulated on the toys like hands. Even so, many of the attributes infants normally encounter in agents were missing: Most of the person was hidden from view, including her face and body, and she never spoke or interacted with the infant. Thus, hiding the actor's skin removed one of a limited set of perceptual attributes, and, given its salience for adults, it may have been a particularly important one for infants.

If infants identify agents based on the weight of the perceptual evidence, then providing more featural support might enable them to understand the gloved reaches. To test this possibility, in Studies 2 and 3 we provided infants with additional information about the gloved hands-highlighting the fact that the hands were part of a person. In Study 2, this information was given prior to the habituation procedure. In Study 3, this information was included as part of the habituation and test events. In each study, all infants were shown gloved reaching events, and the question of interest was whether these additional sources of information supported their interpretation of the gloved grasps as goal directed.

\section{STUDY 2}

\section{Method}

Participants. Forty infants, recruited as in Study 1, participated in Study 2. Twenty infants were 7-month-olds, and 20 infants were 12-month-olds. The 6 girls and 14 boys in the 7-month-old group were between 6 months, 13 days and 7 months, 30 days old ( $M=7$ months, 2 days). The 10 girls and 10 boys in the 12-month-old group ranged in age from 11 months, 0 days to 12 months, 21 days $(M=12$ months, 1 day). Five additional infants began the procedure but were eliminated from the final sample due to failure to complete all trials $(n=2)$ or due to experimenter error $(n=3)$.

Procedure. Prior to the habituation task, each infant participated in a preexposure phase in the waiting room. Wearing gold gloves on both hands, the actor interacted with the infant for a period of $5 \mathrm{~min}$. She handed toys to the infant and manipulated puzzles and other games while attempting to ensure that the infant noticed the gloves. Because we observed individual variation in the extent to which infants seemed to notice the gloves, we subsequently coded the videotape of 
this phase. ${ }^{4}$ An observer who was not aware of the infants' habituation performance coded the number and duration of infants' looks toward the gloves and the number of times infants alternated gaze between the gloves and the actor's face. The first of these indicated the extent to which infants attended to the gloves, and the second served as an index of the extent to which infants related the gloves to the person who wore them. To assess reliability, a second independent observer coded the tapes of 9 infants. The two observers were strongly correlated in their assessments of the average length of look $(r=.99)$ and the number of alternating looks $(r=.84)$.

After the preexposure phase, the actor and infant moved to the habituation room to begin the habituation procedure. The habituation procedure was identical to the gloved hand condition in Study 1. Ten infants at each age saw the actor reach to the right side of the stage during habituation, and 10 saw her grasp the bear during habituation. Ten infants at 7 months and 11 infants at 12 months began the test phase with a new object trial.

As in Study 1, reliability was assessed by having a second observer code each infant from the videotape. The two observers were correlated in their judgments of infants' looking times on the two kinds of test trials $(r=.90)$. The second observer agreed with the live observer on $79 \%$ of the trial endings for 7-month-olds and $92 \%$ of the trial endings for 12-month-olds. The direction of these disagreements was randomly distributed across trial types at both 7 and 12 months, Fisher's exact test, $p>.99$ and $p>.40$, respectively.

\section{Results}

Table 2 summarizes infants' looking times during the habituation procedure. The two age groups did not differ in the number of trials required to meet criterion or in their looking times during habituation. An ANOVA comparing the two age groups on the first and last three habituation trials revealed only a main effect of trial, $F(5$, $190)=12.08, p<.0001$, reflecting a decline in looking across the habituation phase. Five 7-month-olds and one 12-month-old reached 14 habituation trials without meeting the habituation criterion.

Preliminary analyses revealed no reliable effects on infants' differential responding to the two test events based on the sex of the infant, the type of test event presented first, the side to which the actor reached during habituation, or the toy grasped during habituation. Therefore, subsequent analyses collapsed across these dimensions. An ANOVA with age group ( 7 months vs. 12 months) as the between-subjects factor and test trial type (new object vs. new side) as the within-subjects factor yielded no reliable effects. Separate paired comparisons indicated that neither age group differenti-

\footnotetext{
${ }^{4}$ Five infants in the 7-month age group could not be coded due to lost videotape.
} 
TABLE 2

Attention During Habituation Trials and the Three Test Trials of Each Type in Study 2 by Age Group and Level of Monitoring During Preexposure

\begin{tabular}{|c|c|c|c|c|c|c|c|c|c|c|}
\hline & \multirow{3}{*}{\multicolumn{2}{|c|}{$\begin{array}{c}\text { No. of } \\
\text { Habituation } \\
\text { Trials }\end{array}$}} & \multicolumn{8}{|c|}{ Average Looking Time (in Sec) } \\
\hline & & & \multicolumn{4}{|c|}{ Habituation } & \multicolumn{4}{|c|}{ Test Trials } \\
\hline & & & \multicolumn{2}{|c|}{ First 3} & \multicolumn{2}{|c|}{ Last 3} & \multicolumn{2}{|c|}{ New Side } & \multicolumn{2}{|c|}{ New Object } \\
\hline & $M$ & $S E$ & $M$ & $S E$ & $M$ & $S E$ & $M$ & $S E$ & $M$ & $S E$ \\
\hline \multicolumn{11}{|l|}{ Age group } \\
\hline 7 months & 9.9 & 0.7 & 10.2 & 1.3 & 5.6 & 1.0 & 6.8 & 1.4 & 7.1 & 1.0 \\
\hline 12 months & 8.2 & 0.6 & 9.0 & 1.4 & 3.8 & 0.5 & 7.7 & 1.3 & 7.7 & 1.3 \\
\hline \multicolumn{11}{|l|}{ Preexposure } \\
\hline High monitor $^{\mathrm{a}}$ & 8.9 & 1.0 & 8.2 & 1.0 & 3.5 & 0.3 & 4.8 & 0.9 & 7.5 & 1.8 \\
\hline Low monitor ${ }^{b}$ & 8.7 & 0.5 & 10.4 & 1.5 & 5.3 & 0.9 & 8.7 & 1.4 & 7.7 & 1.0 \\
\hline
\end{tabular}

$\mathrm{a}_{n}=11 . \mathrm{b}_{n}=24$.

TABLE 3

Infants' Attention to the Gloved Experimenter During the Preexposure Phase of Study 3

\begin{tabular}{lrrr}
\hline & Range & $M$ & $M d n$ \\
\hline Means length (in sec) of looks to the experimenter's hands & & & \\
$\quad$ Overall & $1.5-23.4$ & 9.2 & 6.6 \\
7 months & $1.5-20.5$ & 8.0 & 5.6 \\
$\quad 12$ months & $4.4-23.4$ & 10.1 & 8.0 \\
No. of gaze shifts between the experimenter's hands and face & & & \\
$\quad$ Overall & $0-16$ & 5 & 4 \\
7 months & $0-14$ & 5 & 2 \\
12 months & $0-16$ & 6 & 4 \\
\hline
\end{tabular}

ated between the two types of test trials, $t(19)=0.02, p>.9$ at 12 months, and $t(19)=$ $0.34, p>.7$ at 7 months. Relative to the last three habituation trials, 12 -month-olds recovered on both new object trials, $t(19)=5.63, p<.0001$, and new side trials, $t(19)=$ $4.59, p<.002$ trials, and 7 -month-olds recovered on neither kind of test trial, $t(19)=$ $1.87, p<.07$ for new object trials, and $t(19)=0.82, p>.4$ for new side trials. These results suggest that prior experience with the gloved experimenter did not strongly impact infants' subsequent responses to the gloved reaching events.

However, infants varied in the extent to which they seemed to notice the gloves during the preexposure phase. Some infants glanced at them only briefly, whereas others stared at them for long periods of time. Some infants repeatedly looked back and forth between the experimenter's gloved hands and face, whereas others seldom or never did this. Table 3 summarizes these responses for the two age groups. 
We therefore next asked whether those infants who were especially attentive to the gloves during the preexposure phase responded more systematically on test trials. Seven- and 12-month-olds differed neither in their average length of glance to the gloves, $t(33)=1.02, p>.31$, nor in the number of gaze alternations between the experimenter's hands and face, $t(33)=0.6, p>.5$. Eleven infants (five 7-month-olds and six 12-month-olds) were above the median on both measures, henceforth called the high-monitoring group. Habituation responses were analyzed for the high-monitoring infants versus the rest of the sample, henceforth called the low-monitoring group (see Table 2). An ANOVA with training status (high vs. low monitoring) as the between-subjects factor and trial type as the within-subjects factor revealed a significant Training Status $\times$ Trial Type interaction, $F(1,33)=$ $4.63, p<.05 .{ }^{5}$ High-monitoring infants tended to look longer on new object trials than on new side trials, $t(10)=2.05, p=.07$, whereas low-monitoring infants did not differentiate between the test events, $t(23)=1.04, p>$.3. Relative to the last three habituation trials, high-monitoring infants recovered on new object trials, $t(10)=2.83, p<.05$, but not on new side trials, $t(10)=1.23, p>.2$. Low-monitoring infants recovered on both kinds of test trials, $t(23)=4.09, p<.005$ for new object trials, and $t(23)=3.35, p<.0005$ for new side trials.

\section{Discussion}

As a group, neither the 7-month-olds nor the 12-month-olds encoded the gloved reaches as being goal directed. This finding reinforces the findings of Study 1 in indicating that disrupting the surface features of the hand disrupted infants' interpretation of the grasping events. Moreover, the findings of Study 2 indicate that, in general, prior experience with the gloved experimenter did not alter infants' responses to the gloved reaching events. However, we found that infants who had closely monitored the gloves during preexposure differed in their responses from infants who did not closely monitor the gloves. This subset of infants showed a trend consistent with their interpreting the gloved reaches as goal directed. It seems as if some infants were able to draw on prior experience to make sense of the gloved hands, but other infants did not pick up on the information they needed during the preexposure. These considerations motivated the third study.

\section{STUDY 3}

In Study 3, we attempted to ensure that infants could readily discern that the gloved hands were attached to a person. To do this, we presented the relevant information

\footnotetext{
${ }^{5}$ When age group was included as a factor in this analysis, it yielded no reliable effects.
} 
during the experimental events. Throughout the procedure, infants viewed the gloved experimenter from the waist up and could therefore readily observe that the gloved hands were part of a person. Given this configuration, infants viewed events in which the actor's gaze was coordinated with her gloved grasp. In interpreting the findings, therefore, we anticipated needing to consider how infants' knowledge about gaze might have contributed to their responses. With this need in mind, we tested infants at three ages-7, 9, and 12 months - to parallel the ages at which prior work had investigated infants' sensitivity to the object directedness of gaze (Woodward, 2003).

\section{Method}

Participants. Sixty infants, recruited as in Studies 1 and 2, participated in Study 3. Twenty infants were tested at each of three ages: 7, 9, and 12 months. The 7-month-olds ranged in age from 6 months, 0 days to 8 months, 0 days $(M=7$ months, 4 days); the 9-month-olds ranged in age from 8 months, 0 days to 10 months, 1 day ( $M=8$ months, 26 days); and the 12-month-olds ranged in age from 11 months, 7 days to 12 months, 24 days ( $M=12$ months, 0 days). There were 7 girls and 13 boys each in the 7- and 9-month age groups, and 10 girls and 10 boys in the 12-month age group. An additional 9 infants began the procedure but were excluded from the final sample due to failure to complete all trials (two 7-montholds and two 12-month-olds) or due to experimenter error (one 7-month-old and three 9-month-olds).

Procedure. As in Studies 1 and 2 a visual habituation paradigm was used. However, instead of the actor reaching in from offstage, she sat behind and between the two toys, with her upper body and face visible to the infant (see Figure 2 ). The actor wore a red long-sleeved top and metallic gold gloves on both hands. At the beginning of each trial, the actor made eye contact with the infant and said "Hi, look!" as she looked at and grasped one of the toys. She then remained still, looking at and holding the toy until the trial ended. Infants saw the actor grasp the same toy in the same location on each habituation trial. Ten infants at 7 months, 11 infants at 9 months, and 9 infants at 12 months saw the actor reach to the right side of the stage. Ten infants at each age saw the actor grasp the bear. As in the first two studies, infants' looking was timed beginning when the actor stopped moving in contact with the toy and ending when the infant looked away for 2 consecutive sec. The habituation criterion was computed as in Studies 1 and 2.

Following habituation, the positions of the toys were reversed for test trials. In contrast to Studies 1 and 2, there was no familiarization trial between the habituation and the test phases because having the actor in the display made this trial awkward. In the test trials, each infant saw three new object and three new side trials in 


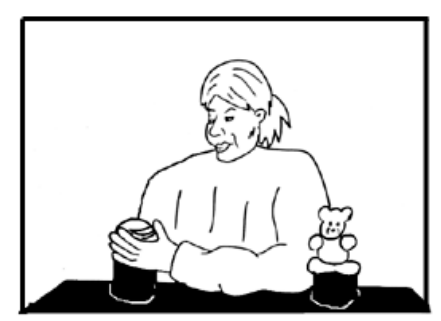

Habituation Event

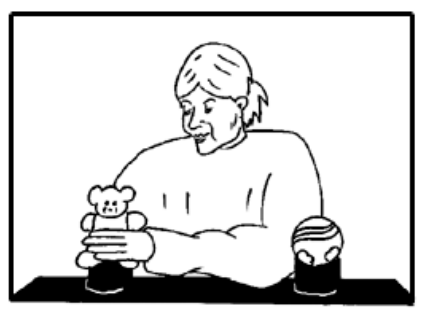

New-Object Event

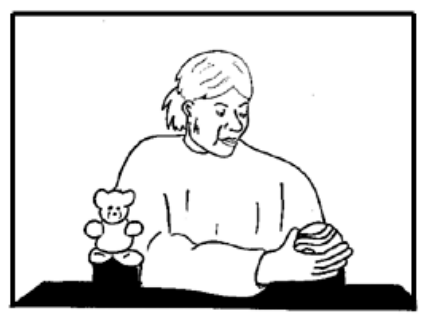

New-Side Event

FIGURE 2 Sample habituation and test events for Experiment 3.

alternation. Eleven infants at 7 months and 10 each at 9 and 12 months began with a new object trial.

As in Studies 1 and 2, reliability was assessed by having a second observer code each infant from the videotape. The two observers' judgments of looking times were strongly correlated $(r=.92)$. The second observer agreed with the live observer on the endpoints of $84 \%$ of trials for 7 -month-olds, $81 \%$ for 9 -month-olds, and $84 \%$ for 12 -month-olds. The disagreements were distributed randomly across trial types for each group, Fisher's exact $p>.99, p>.07$, and $p>.28$ at 7, 9 , and 12 months, respectively.

\section{Results}

Table 4 summarizes infants' looking times. As in Studies 1 and 2, infants' looking times during the first and last three habituation trials were entered into a repeated measures ANOVA with age group as the between-subjects factor. This analysis revealed a main effect of trial, $F(5,285)=46.52, p<.0001$, and no reliable age effects. There were no reliable age differences in the number of habituation trials required to meet criterion. Two infants each at 7 and 9 months and 1 infant at 12 months reached 14 habituation trials without meeting the criterion. 
TABLE 4

Attention During Habituation Trials and the Three Test Trials of Each Type for Each Age Group in Study 3

\begin{tabular}{|c|c|c|c|c|c|c|c|c|c|c|}
\hline \multirow[b]{4}{*}{ Age Group } & & & \multicolumn{8}{|c|}{ Average Looking Time (in Sec) } \\
\hline & \multirow{2}{*}{\multicolumn{2}{|c|}{$\begin{array}{c}\text { No. of } \\
\text { Habituation } \\
\text { Trials }\end{array}$}} & \multicolumn{4}{|c|}{ Habituation } & \multicolumn{4}{|c|}{ Test Trials } \\
\hline & & & \multicolumn{2}{|c|}{ First 3} & \multicolumn{2}{|c|}{ Last 3} & \multicolumn{2}{|c|}{ New Side } & \multicolumn{2}{|c|}{ New Object } \\
\hline & $M$ & $S E$ & $M$ & $S E$ & $M$ & $S E$ & $M$ & $S E$ & $M$ & $S E$ \\
\hline 7 months & 8.5 & 0.7 & 23.9 & 3.6 & 7.7 & 1.7 & 8.8 & 1.3 & 9.7 & 1.7 \\
\hline 9 months & 8.4 & 0.6 & 24.3 & 7.7 & 7.7 & 1.4 & 10.4 & 1.7 & 13.7 & 2.5 \\
\hline 12 months & 8.1 & 0.6 & 13.3 & 1.5 & 5.0 & 0.4 & 6.5 & 0.8 & 8.7 & 0.7 \\
\hline
\end{tabular}

Preliminary analyses revealed no effects of the toy grasped during habituation, the side reached to in habituation, or the test event presented first. Therefore, subsequent analyses collapsed across these factors. An ANOVA with age group (7, 9, or 12 months) as the between-subjects factor and trial type (new object vs. new side) as the within-subjects factor revealed a main effect of trial type, $F(1,57)=$ $6.90, p<.01$, indicating longer looking times on new object trials than on new side trials. The analysis did not indicate that this pattern varied across age groups: Neither the main effect of age, $F(1,57)=2.62, p>.08$, nor the Age $\times$ Condition interaction, $F(1,57)=0.70, p>.5$, reached significance. ${ }^{6}$

Although the ANOVA did not indicate reliable age differences, separate analyses were conducted to test whether each age group reflected the overall pattern of longer looking times on new object trials than on new side trials. ${ }^{7}$ These analyses indicated that whereas 12- and 9-month-olds looked reliably longer on new object trials than on new side trials, $t(19)=4.23, p<.005$ at 12 months, and $t(19)=2.78, p<.05$ at 9 months, this pattern was not reliable at 7 months, $t(19)$ $=0.26, p>$.7. Excluding those infants who did not reach the habituation criterion did not change these results. Compared to the final three habituation trials, 12 -month-olds recovered on new object trials, $t(19)=6.60, p<.0001$, but not on new side trials, $t(19)=1.79, p>.09 ; 9$-month-olds recovered on both new object trials, $t(19)=5.00, p<.0001$, and new side trials, $t(19)=2.52, p>.05$; and 7 -month-olds recovered on neither new object trials, $t(19)=1.73, p>.1$, nor new side trials, $t(19)=1.43, p>.1$.

\footnotetext{
${ }^{6}$ The data from the 9-month-olds had positive skew. When the data were log transformed to reduce this skew, the results of the Age Group $\times$ Trial Type ANOVA were similar to those reported for the untransformed scores: There was a main effect of trial type, $F(1,57)=13.79, p<.0005$, with neither the main effect of age group, $F(2,57)=2.46, p>.09$, nor the Age Group $\times$ Trial Type interaction, $F(2,57)=$ $2.64, p>.08$, reaching significance.

${ }^{7}$ The data for the 9-month-olds were first log transformed to correct for skew.
} 
Further analyses indicated a sex difference at 7 months, but not at the other two ages: A Sex $\times$ Trial Type ANOVA conducted at 7 months revealed a Sex $\times$ Trial Type interaction, $F(1,18)=9.83, p<.01$. Follow-up analyses revealed that 7-month-old girls looked longer on new object $(M=12.3, S E=3.6)$ than new side $(M=7.5, S E=2.2)$ trials, $t(6)=2.68, p<.05$, but that 7-month-old boys did not differentiate between new object $(M=8.3, S E=1.8)$ and new side $(M=9.5, S E=1.6)$ trials, $t(12)=1.14, p>.2$.

\section{Discussion}

When infants observed that the gloved hands were part of a person, they responded to the goal-directed structure of the gloved reaches. This finding indicates that rather than responding rigidly based on the presence of perceptual triggers, infants draw flexibly on information in the context to interpret potentially ambiguous events. Together with the findings from Study 2, these results suggest that infants do this more readily when the relevant information accompanies the ambiguous event rather than precedes it.

The findings of Study 3 also suggest a possible age difference in infants' responses. Although the main analysis did not indicate reliable age differences, when each age group was analyzed separately, an age difference emerged: The older two age groups responded to the change in the actor-object relation, but as a group, the youngest infants did not respond in this way. Subsequent analyses suggested that this pattern at 7 months may have been due only to the boys; girls at this age responded as did the older infants. However, given the relatively small number of girls $(n=7)$ tested at this age, further studies are necessary to confirm this sex difference.

Why might the youngest have been less sensitive than the older infants to the object directedness of the gloved reaches? One possibility is that the experimental events were in some way distracting for the 7-month-olds. Perhaps having the actor's face visible led infants to attend more to the face and less to the hands, thus detracting attention from the experimentally relevant aspects of the event. Recent findings argue against this possibility: When 7-month-olds viewed identical events in which the actor's hands were bare they responded by looking longer on new object trials than on new side trials, and there was no sex difference in responding in that study (Woodward, 2003). A second possibility is that the 7-month-olds were less able than older infants to integrate information about the actor as a whole. They may have noticed the gloved hands but have been less able than older infants to recruit information about the rest of the actor to interpret her actions.

The inclusion of gaze in the experimental events raises the question of whether the findings for 9- and 12-month-olds could be due to gaze alone. Perhaps infants ignored the odd hands, focusing only the actor's gaze. If infants understood gaze as an object-directed action, then this alone might be sufficient to drive the effect. 
Several recent findings suggest that this explanation cannot account for all of the current findings. To start, when 9-month-olds view gaze alone or gaze accompanied by a pointing gesture, they follow the actor's line of regard, but do not seem to encode the actor-object relation (Woodward, 2003; Woodward \& Guajardo, 2002). Thus, it is unlikely that gaze alone could have driven the effect at 9 months. In contrast, 12-month-old infants do respond to the object-directed structure of gaze (Woodward, 2003). However, infants at this age do not ignore the manual actions that accompany gaze. When gaze accompanies causally implausible actions, 12-month-olds do not respond based on gaze alone (Sommerville \& Woodward, in press; Woodward \& Sommerville, 2000).

\section{GENERAL DISCUSSION}

In the first study, we asked whether disrupting the typical surface features of a human hand would influence infants' sensitivity to the goal directedness of its actions. Replicating prior results, we found that when 7- and 12-month-old infants viewed a bare hand reach through a curtain to grasp a toy, they encoded this event in terms of the relation between the hand and its goal. However, when infants viewed the same events with a gloved hand, they did not respond in this way. The gloved and bare hand events were identical on many dimensions, including the motion of the actor's hand and the way in which the hand articulated on the object. Nevertheless, infants interpreted the gloved and bare hand events differently. Because infants' overall levels of looking did not differ in the two conditions, this finding seems not to be due to infants having a strong preference for or aversion to the gloved hand relative to the bare hand. Studies 2 and 3 each provided evidence that additional information about the gloved hands, in particular, the opportunity to view the actor who wore the gloves, supported infants' interpretation of the gloved reaches as goal directed.

Several kinds of motion have been proposed to support infants' interpretation of events as goal directed, including biological patterns of motion (Baron-Cohen, 1995), motion around barriers in pursuit of goals (e.g., Gergely et al., 1995), contingent responding to the actions of social partners (S. Johnson et al., 1998; Kuhlmeier et al., 2003b), and self-propelledness (Premack, 1990). None of these on its own accounts for the current findings. Biological motion was present in each of the experimental events, and motion around barriers was present in none of them. Therefore these features cannot account for infants' differential responding across studies and conditions. There was no information about social contingency in the events for Study 1 and minimal contingency information in Study $3 .^{8}$ There-

\footnotetext{
${ }^{8}$ Although the actor greeted the infant at the start of each trial, she did not engage in sustained contingent responding to the infant's actions.
} 
fore, this factor cannot account for infants' differential responding to the gloved and bare hand reaches. Information about self-propelledness varied across the experiments but nevertheless seems unlikely to account for the findings. For one, the gloved and bare hand events in Study 1 were equally ambiguous with respect to the self- propelledness of the arm and nevertheless infants responded differentially to them. In addition, other recent findings indicate that self-propelledness is neither sufficient (Shimizu \& Johnson, 2004) nor necessary (Csibra et al., 1999) to support goal attribution in older infants.

Taken together, these findings indicate that surface features are relevant to infants' determination that an entity is an agent, thereby calling into question the claim that motion properties provide the sole means by which infants identify goal-directed agents. However, we do not conclude from these findings that self-propelled motion or other patterns of motion are unimportant sources of information for infants. Nor do we conclude that familiar surface features must be present for infants to identify an object as a potential agent. Indeed, the results of Studies 2 and 3 indicated that infants were sometimes able to interpret the gloved reaches as goal directed when given the chance to observe more of the actor who wore the gloves. Rather, we conclude that infants, like adults, draw on their knowledge of the rich perceptual attributes of agents when making sense of novel events. These attributes include not only patterns of motion but also typical surface features.

A potential alternative explanation for these findings is that the glove did not disrupt infants' determination that the hand was part of an agent but instead disrupted their ability to identify the grasp as a goal-directed action. Perhaps infants identified the gloved hands as parts of a person but were uncertain what the purpose of the gloved grasp was. Consistent with this possibility, several studies have found that infants sometimes fail to represent unfamiliar hand gestures as goal directed (Woodward, 1999; Woodward \& Guajardo, 2002; but see Jovanovic et al., 2003; Király et al., 2003). In this case, the prior experience with the gloved actor in Study 2 and the additional information provided by the fully visible actor in Study 3 might have clarified the goal of the action rather than the identity of the actor.

This possibility is consistent with the general conclusion that infants' initial action knowledge is not wholly abstract but instead is constrained by their knowledge of real agents and their actions. However, although it is possible that confusion about the action contributed to infants' responses in the gloved condition, we do not believe that this explanation fully accounts for the findings. For one, we find it significant that infants' propensity to alternate gaze between the actor's face and hands in Study 2 was related to their subsequent interpretation of the gloved reaches as goal directed. This suggests that integrating information about the agent mattered for infants' responses. Moreover, the additional behavioral evidence provided in Study 3 has not been effective in leading infants to interpret ambiguous actions as goal directed in other studies. Specifically, when 9- through 12-month-old infants observe 
gaze and posture shifts that are coordinated with unfamiliar or ambiguous manual actions, they do not interpret these actions as goal directed (Sommerville \& Woodward, in press; Woodward \& Sommerville, 2000). Therefore, we conclude that although confusion about the action itself may have contributed to infants' responses to the gloved reach, confusion about the nature of the agent also contributed to these responses.

We turn now to considering how these findings relate to the literature, reviewed earlier, on infants' interpretation of unusual agents, beginning with the findings for 7-month-olds. The finding that 7-month-olds did not readily respond to the gloved events as goal directed is quite consistent with the existing literature. Studies testing 5- to 7-month-old infants have shown that they are sensitive to the goal structure of certain human actions when they are performed by bare-handed actors, consistent with the findings of Study 1 (Jovanovic et al., 2003; Woodward, 1998, 2003). However, when infants at these ages have been tested with computer-generated shapes or inanimate agents, they have not responded systematically (Csibra et al., 1999; Jovanovic et al., 2003; Woodward, 1998). Studies that find that infants respond to novel entities as agents have tested older infants, mainly at 12 months or older (Gergely et al., 1995; S. Johnson et al., 2001; S. Johnson et al., 1998; Kuhlmeier et al., 2003b; Shimizu \& Johnson, 2004), although there are two reports of infants as young as 9 months showing this kind of response (Csibra et al., 1999; Kuhlmeier, Wynn, \& Bloom, 2003a). Taken together, these findings suggest that infants' initial sensitivity to the goal structure of action is tightly linked to the most typical agents in their environment, that is, humans.

In contrast, these findings for 12-month-olds appear to be inconsistent with several other findings. Whereas the results of Studies 1 and 2 indicate that 12-montholds are limited in their ability to interpret unusual entities as agents, other studies report that 12-month-olds respond to unusual entities as if they were agents given certain kinds of behavioral evidence; for example, when objects move around apparent barriers to reach goals (Csibra et al., 2003; Csibra et al., 1999; Gergely et al., 1995), when they engage in apparent attempts to help or hinder the movement of another object (Kuhlmeier et al., 2003b), or when they participate in contingent social interactions (S. Johnson et al., 2001; S. Johnson et al., 1998; Shimizu \& Johnson, 2004). These considerations suggest that by the end of the first year of life, infants possess relatively abstract knowledge about how agents behave and that, in some cases, this knowledge informs infants' detection of agents. Thus, one possible reason for the disparity in findings may be the different kinds of behavioral evidence present in the experimental events. The events in these studies did not show infants gloved hands that avoided obstacles or engaged in social behavior. Perhaps if they had, the 12-month-old infants would have interpreted their actions as goal directed. Further studies are needed to test this possibility.

However, these findings, along with other recent findings, make clear that 12-month-olds do not depend solely on patterns of motion to identify agents. To 
start, the fact that 12-month-olds interpreted the bare-handed grasps as goal directed indicates that agent detection can occur in the absence of obstacle avoidance, helping behaviors, or contingent responding. In addition, the fact that the glove disrupted 12-month-olds' responding in Studies 1 and 2 indicates that these infants recruit information about surface attributes to identify potential agents. Moreover, other studies report that adding surface features typical of familiar agents to a novel entity can lead older infants to treat it as an agent. For example, Johnson and her colleagues (S. Johnson et al., 2001; S. Johnson et al., 1998) have found that in addition to contingent responsiveness, facial features contribute to 12- and 15-month-old infants' propensity to respond to unusual entities as if they were agents. Consistent with this finding, Kuhlmeier et al. (2003a) reported that 9-month-old infants responded to animated geometric figures as if they were agents when the figures had faces but not when they lacked faces.

Taken together, these findings suggest that infants' action knowledge is initially constrained by the details of particular agents and their actions and becomes more abstract and flexible with development. There are at least two conclusions that can be drawn from these findings. The first is that infants possess abstract, innate conceptions of agents and intentional action but that this knowledge is only expressed as infants discover the real-world cases that embody these principles. This is analogous to the argument that children are born with innate grammars for language that are only evident once they have acquired the linking rules that specify how this grammar is embodied in natural language. If this were the case, these findings would reflect the gradual emergence of the "linking rules" rather than the gradual emergence of abstract knowledge.

To our mind, a compelling alternative is that abstract action knowledge is constructed in development from concrete beginnings. This more abstract knowledge may then provide a basis for infants' interpretation of events in which novel entities act like agents. This account, which posits abstract knowledge as the product rather than the foundation of development is analogous to recent suggestions that grammar arises from similar processes (Tomasello, 2001). As is the case for grammar, several kinds of cognitive processes seem likely to contribute to the construction of action knowledge, including statistical learning (Baldwin \& Baird, 2001), associative learning (Rakison \& Poulin-Dubois, 2001), and structure mapping (Gentner \& Medina, 1998). Moreover, infants' emerging agentive experience may contribute to the development of action knowledge (Meltzoff, 2002; Sommerville \& Woodward, in press; Sommerville, Woodward, \& Needham, in press). The pursuit of these possibilities is a critical direction for future investigation.

\section{CONCLUSION}

We began by noting that in mature systems, knowledge about the typical attributes of real-world agents travels with and informs reasoning about their abstract attrib- 
utes. It has been argued that these two aspects of mature knowledge do not emerge together in ontogeny. Specifically, it has been proposed that infants begin with abstract systems for detecting intentions that are not initially linked to knowledge about real-world agents. The findings reported here call these proposals into question. Across three studies, we found that 7- to 12-month-old infants drew on the surface attributes of real-world agents in determining whether an observed action is goal directed. These findings raise the possibility that infants' experiences with (and perhaps as) real-world agents play a formative role in the development of intentional action knowledge.

\section{ACKNOWLEDGMENTS}

This research was supported by National Institutes of Health Grant R29HD35707-01 and by a Robert R. McCormick Tribune Foundation grant to Amanda L. Woodward. We thank Anneliese Hahn, Monique Duwell, Stacey Hodgins, and Elizabeth Perkowski for their assistance in completing the studies as well as Susan Levine, Camille Wilson-Brune, Jennifer Sootsman, David Rakison, and two anonymous reviewers for their comments on earlier versions of this article. We are indebted to the parents and infants who volunteered their time to participate in the studies.

\section{REFERENCES}

Baldwin, D. A., \& Baird, J. A. (2001). Discerning intentions in dynamic human action. Trends in Cognitive Sciences, 5, 171-178.

Baron-Cohen, S. (1995). Mindblindness: An essay on autism and theory of mind. Cambridge, MA: MIT Press.

Bertenthal, B. J. (1993). Infants' perceptions of biomechanical motions: Intrinsic image and knowledge-based constraints. In C. E. Granrud (Ed.), Visual perception and cognition in infancy (pp. 175-214). Hillsdale, NJ: Lawrence Erlbaum Associates, Inc.

Csibra, G., Biro, S., Koos, O., \& Gergely, G. (2003). One-year-old infants use teleological representations of actions productively. Cognitive Science, 27, 111-133.

Csibra, G., Gergely, G., Biro, S., Koos, O., \& Brockbank, M. (1999). Goal attribution without agency cues: The perception of "pure reason" in infancy. Cognition, 72, 237-267.

Gentner, D., \& Medina, J. (1998). Similarity and the development of rules. Cognition, 65, 263-297.

Gergely, G., Nadasdy, Z., Csibra, G., \& Biro, S. (1995). Taking the intentional stance at 12 months of age. Cognition, 56, 165-193.

Johnson, M. (1992). Imprinting and the development of face recognition: From chick to man. Current Directions in Psychological Science, 1, 52-55.

Johnson, S., Booth, A., \& O'Hearn, K. (2001). Inferring the goals of a nonhuman agent. Cognitive Development, 16, 637-656.

Johnson, S., Slaughter, V., \& Carey, S. (1998). Whose gaze will infants follow? The elicitation of gaze-following in 12-month-olds. Developmental Science, 1, 233-238.

Jovanovic, B., Király, I., Elsner, B., Gergely, G., Prinz, W., \& Aschersleben, G. (2003). The role of effects for infants' perception of action goals. Manuscript submitted for publication. 
Király, I., Jovanovic, B., Prinz, W., Aschersleben, G., \& Gergely, G. (2003). The early origins of goal attribution in infancy. Consciousness and Cognition, 12, 752-769.

Kosugi, D., \& Fujita, K. (2002). How do 8-month-old infants recognize causality in object motion and that in human action? Japanese Psychological Research, 44, 66-78.

Kuhlmeier, V., Wynn, K., \& Bloom, P. (2003a). Attribution of dispositional states by 9-month-olds: The role of faces. Manuscript submitted for publication.

Kuhlmeier, V., Wynn, K., \& Bloom, P. (2003b). Attribution of dispositional states by 12-month-olds. Psychological Science, 14, 402-408.

Legerstee, M., Barna, J., \& DiAdamo, C. (2000). Precursors to the development of intention at 6 months: Understanding people and their actions. Developmental Psychology, 36, 627-634.

Meltzoff, A. N. (2002). Elements of a developmental theory of imitation. In A. N. Meltzoff \& W. Prinz (Eds.), The imitative mind: Development, evolution, and brain bases (pp. 19-41). Cambridge, England: Cambridge University Press.

Molina, M., Van de Walle, G., Condry, K., \& Spelke, E. (in press). The animate-inanimate distinction in infancy: Developing sensitivity to constraints on human actions. Journal of Cognition and Development.

Pinto, J. (1994). MacXhab (Version 1.3). Stanford, CA.

Poulin-Dubois, D., Lepage, A., \& Ferland, D. (1996). Infants' concept of animacy. Cognitive Development, 11, 19-36.

Premack, D. (1990). The infant's theory of self-propelled objects. Cognition, 36, 1-16.

Rakison, D. H., \& Poulin-Dubois, D. (2001). Developmental origin of the animate-inanimate distinction. Psychological Bulletin, 127, 209-228.

Shimizu, Y. A., \& Johnson, S. C. (2004). Infants' attribution of a goal to a morphologically unfamiliar agent. Developmental Science, 7, 425-430.

Smith, L. B., \& Heise, D. (1992). Perceptual similarity and conceptual structure. In B. Burns (Ed.), Percepts, concepts and categories (pp. 233-272). Amsterdam: Elsevier Science.

Sommerville, J. A., \& Woodward, A. L. (in press). Pulling out the intentional structure of action: The link between action production and action processing in infancy. Cognition.

Sommerville, J. A., Woodward, A. L., \& Needham, A. (in press). Action experience alters 3-month-old infants' perception of others' actions. Cognition.

Spelke, E. (1976). Infants' intermodal perception of events. Cognitive Psychology, 8, 553-560.

Spelke, E. S., Phillips, A. T., \& Woodward, A. L. (1995). Infants' knowledge of object motion and human action. In A. J. Premack, D. Premack, \& D. Sperber (Eds.), Causal cognition: A multidisciplinary debate (pp. 44-77). Oxford, England: Clarendon.

Tomasello, M. (2001). Do young children have adult syntactic competence? Cognition, 74, 209-253.

Woodward, A. L. (1998). Infants selectively encode the goal object of an actor's reach. Cognition, 69, $1-34$.

Woodward, A. L. (1999). Infants' ability to distinguish between purposeful and non-purposeful behaviors. Infant Behavior and Development, 22, 145-160.

Woodward, A. L. (2003). Infants' developing understanding of the link between looker and object. Developmental Science, 6, 297-311.

Woodward, A. L., \& Guajardo, J. J. (2002). Infants' understanding of the point gesture as an object-directed action. Cognitive Development, 17, 1061-1084.

Woodward, A. L., \& Sommerville, J. A. (2000). Twelve-month-old infants interpret action in context. Psychological Science, 11, 73-77.

Woodward, A. L., Sommerville, J. A., \& Guajardo, J. J. (2001). How infants make sense of intentional action. In B. Malle, L. Moses, \& D. Baldwin (Eds.), Intentions and intentionality: Foundations of social cognition (pp. 149-169). Cambridge, MA: MIT Press. 
Copyright of Infancy is the property of Lawrence Erlbaum Associates and its content may not be copied or emailed to multiple sites or posted to a listserv without the copyright holder's express written permission. However, users may print, download, or email articles for individual use. 